ist im übrigen ein reichhaltiger. Vergleicht man es mit dem Salmon'schen Lehrbuche, so wird vor allem die eingehende Lehre von der Kurvendiskussion ins Auge fallen. Auch die Theorie der Kurven dritter Ordnung ist recht ausführlich behandelt, wenngleich die Formentheorie nicht in Betracht gezogen. wird. Die Einteilung in Gattungen wird nach Wiener und Köhnel vorgetragen und die Beziehungen zwischen der Hesse'schen und Cayley'schen Kurve, der Grundkurve und der ihr assoziierten Kurve dritter Klasse sind in einfacher Weise dargestellt. Die Grundzüge einer Gestaltsuntersuchung der Kurven vierter Ordnung sind gleichfalls aufgenommen, daneben viele Einzelheiten z. B. Czubers Methode der Enveloppenbestimmung. Zahlreiche passende Beispiele sind eingeschaltet. Eine gewisse Frische der Darstellung ist ein Vorzug des Buches, der für den Lernbeflissenen besonders ins Gewicht fällt. $G . K$.

Bases physiques de la Musique. Par M. H. Bou as e, professeur a la faculté des sciences de Toulouse. ("Scientia", série physico-mathématique $\mathbf{n}^{0}$ 28) 109 p., $8^{0}$ écu. Paris, GauthierVillars, 1906. cart. Fes. 2.-.

Das vorliegende Bändchen enthält in kurzer und übersichtlicher $\mathrm{Zu}$ sammenfassung das Wissenswerteste über die physikalischen Grundlagen der Musik unter Zugrundelegung der Helmholtz'schen Theorie. Wenn auch neue Resultate wenig Berücksichtigung fanden, ist das Büchlein doch als eine vortreffliche Einführung in die musikalische Akustik zu empfehlen.

\title{
P. Ernst.
}

Kurzes Lehrbuch der Festigkeitslehre. Von Dr. E. Glinzer. Für Baugewerkschule und Baupraxis. Dritte, vielfach umgearbeitete und vermehrte Auflage $\nabla 0 m$,Grundriß der Festigkeitslehre". Leipzig, H. A. Ludwig Degener, 1907.

Dieses Buch ist den Bedürfnissen der staatlichen Baugewerkschule in Hamburg entsprossen. Die Anstalt erhielt in neuester Zeit eine Tiefbauabteilung, so daß eine Umarbeitung der zweiten Auflage der Sammlung vom Jahre 1898 bedingt wurde. So hat das Büchlein, das 1890 als „Grundriß der Festigkeitslehre" zum ersten Male erschien, schon eine ansehnliche Tradition.

Dr. Glinzer bespricht in leicht faßlicher Weise die Grundzüge der technischen Mechanik und Materialienkunde in einer Weise, die es dem Leser ermöglicht, die Formeln unmittelbar anzuwenden. Ausgehend von den Begriffen Festigkeit, Elastizität, Elastizitätsgrenze u. s, w, werden in den einzelnen Absehnitten die Zug-, Druck-, Schub-, Biegungs-, Drehungs-, Knickfestigkeit und die ainfachsten Fälle der zusammengesetzten Beanspruchung, der exzentrische Zug und Druck, beśprochen. Ein Muster für eine einfache statische Berechnung und eine als Anhang beigegebene Tabellensammlung beschließen die Ausführungen. Jedem Abscinitte sind erschöpfende, der subalternen Baupraxis entnommene Ŭbungsbeispiele hinzugefügt. - Wir bezweifeln nicht, daB das Buch den Anforderungen vollkommen entspricht, die Ingenieur und Architekt an Maurer- und Zimmermeister, an den Techniker überhaupt zu stellen gewohnt sind. 